This item was submitted to Loughborough's Research Repository by the author.

Items in Figshare are protected by copyright, with all rights reserved, unless otherwise indicated.

\title{
Decoding gas-solid interaction effects on adsorption isotherm shape: I. Non- polar adsorptives
}

PLEASE CITE THE PUBLISHED VERSION

https://doi.org/10.1016/j.micromeso.2018.01.010

\section{PUBLISHER}

(C) Elsevier

VERSION

AM (Accepted Manuscript)

\section{PUBLISHER STATEMENT}

This work is made available according to the conditions of the Creative Commons Attribution-NonCommercialNoDerivatives 4.0 International (CC BY-NC-ND 4.0) licence. Full details of this licence are available at: https://creativecommons.org/licenses/by-nc-nd/4.0/

\section{LICENCE}

CC BY-NC-ND 4.0

\section{REPOSITORY RECORD}

Madani, S. Hadi, Philip Kwong, Francisco Rodriguez-Reinoso, Mark J. Biggs, and Phillip Pendleton. 2019. "Decoding Gas-solid Interaction Effects on Adsorption Isotherm Shape: I. Non-polar Adsorptives". figshare. https://hdl.handle.net/2134/28228. 


\section{Decoding gas-solid interaction effects on adsorption}

\section{isotherm shape: I. Non-polar adsorptives}

S. Hadi Madani ${ }^{a, b}$, Phillip Kwong ${ }^{c}$, Francisco Rodríguez-Reinoso ${ }^{d}$, Mark J. Biggs ${ }^{c, e}$, Phillip Pendleton ${ }^{\text {c\# }}$

${ }^{a}$ Ian Wark Research Institute, University of South Australia, Adelaide, SA 5001, Australia

${ }^{b}$ Australian School of Petroleum, The University of Adelaide, Adelaide, SA 5005, Australia

c School of Chemical Engineering, The University of Adelaide, Adelaide, SA 5005, Australia.

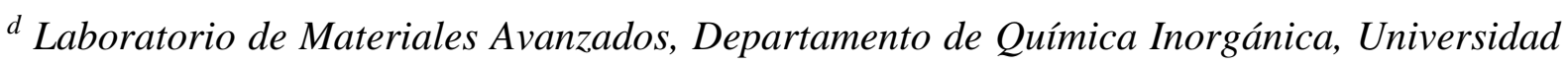
de Alicante. Apartado 99; E-03080, Spain.

e School of Science, Loughborough University, Leicestershire, LE11 3TU, UK

" Corresponding Author: E-mail: Phillip.Pendleton@adelaide.edu.au; Tel: +61 (0)8 83131265

KEYWORDS: adsorption, non-specific interaction, adsorbate, adsorbent, mechanism, polarity, polarizability 


\section{ABSTRACT}

A suite of non-polar adsorptives of different kinetic diameters and shape were used to determine adsorption and pore filling mechanism of a well-characterised poly(furfuryl alcohol)-based activated carbon. Triplicate measured Type I adsorption isotherms for each adsorptive were averaged to provide standard deviation in relative pressures and associated amounts adsorbed. Plateau amounts adsorbed for $\mathrm{N}_{2}$, Ar, $\mathrm{CH}_{4}$, and $\mathrm{C}_{6} \mathrm{H}_{6}$, provided Gurvitsch volumes averaged to $0.368 \pm 0.015 \mathrm{~cm}^{3}(\mathrm{liq}) / \mathrm{g}$. The calculated Gurvitsch volumes were compared with those derived via the Dubinin-Radushkevich (DR) equation. Additional adsorptives were $\mathrm{CO}_{2}$, iso-butane and $\mathrm{SF}_{6}$. The results of these 7 adsorptives were used to qualitatively analyse and decode a micropore filling adsorption mechanism. The DR equation was also used for further analysis of the pore filling mechanism. Based on the adsorbate-adsorbate and adsorbate-adsorbent interactions, adsorbates were classified into three groups: (a) Non-polar with nonspecific interactions (no dipole, no quadrupole, not readily polarizable: $\mathrm{Ar}, \mathrm{N}_{2}, \mathrm{CH}_{4}$ and iso-butane), adsorbing as a continuous uptake over the observed relative pressure range; (b) Non-polar adsorptives with potential for specific interactions (no dipole, quadrupole moment: $\mathrm{CO}_{2}$ and $\mathrm{C}_{6} \mathrm{H}_{6}$ ), adsorbing as a condensation process over a relatively narrow relative pressure range in a medium pressure range; (c) Halogenated adsorptives (no dipole, no quadrupole, polarizable: $\mathrm{SF}_{6}$ ), adsorbing with an S-shaped uptake extending over a relatively broad relative pressure range. 


\section{INTRODUCTION}

Preparation, activation, modification, and application of porous carbonaceous materials are the subjects of a variety of researches over the past few decades. Characterization of these materials using gas adsorption techniques is a key step in their application ${ }^{[1]}$. Kiselev classified adsorptives as two distinct types: non-polar and polar with various sub-categories due to adsorptive chemical properties ${ }^{[2]}$. Gregg and Sing summarised these, directing non-polar adsorptives towards adsorbent structure characterisation and polar adsorptives towards surface chemistry analyses. Maximum information from the latter relies on detailed interpretation of the former ${ }^{[3]}$. A gap exists in the literature whereby no systematic or detailed study has been reported examining the mechanism of adsorption via various molecules that could provide insight into adsorbent structure and chemistry effects on adsorbateadsorbent interactions and adsorbate-adsorbate interactions.

The experimental practicalities of liquid nitrogen $\left(77 \mathrm{~K}\right.$ ) have seen $\mathrm{N}_{2}$ become the molecule most widely used for adsorbent structure analysis ${ }^{[1]}$. Its relatively small kinetic diameter of $0.36 \mathrm{~nm}$ promotes analysis of micropores and its ready condensation below saturation pressure conditions promotes mesopores analysis. Consequently, $\mathrm{N}_{2}$ adsorption isotherms are principal for standard adsorption data for isotherm analyses ${ }^{[4]}$ and pore size distribution (PSD) modelling ${ }^{[5]}$. The low adsorption temperature and kinetic dimension contribute to diffusion limitations to $\mathrm{N}_{2}$ adsorption equilibrium in pores identified as ultra-micropores, widths $\leq 0.7 \mathrm{~nm}$. An alternative adsorptive for these pores is carbon dioxide, which offers a smaller kinetic diameter, $0.33 \mathrm{~nm}$, and adsorption isotherm temperature typically at $273 \mathrm{~K}$, reducing diffusion barriers. In comparison with $\mathrm{N}_{2}, \mathrm{CO}_{2}$ has the disadvantage of a relatively high quadrupole moment making it sensitive to surface polar groups; its high saturation vapour pressure at $273 \mathrm{~K}$ dramatically narrows the applicable relative pressure range (under 1 bar pressure limitations), nullifying mesoporosity characterization ${ }^{[6]}$. Appropriate analysis of adsorption isotherms due to both adsorptives would provide a complementary picture of all micropores and mesopores ${ }^{[7]}$. In contrast to the shape and modest surface chemistry susceptibility of these two adsorptives, Ar is a non-polar, spherical adsorptive of intermediate kinetic diameter, which has received support as an alternative to $\mathrm{N}_{2}$; Recent IUPAC recommendations suggest Ar to be the preferred adsorptive ${ }^{[8]}$. Argon also suffers from the low temperature diffusion problems of $\mathrm{N}_{2}$ for small micropores ${ }^{[9]}$ and, liquid argon is not readily available to all experimentalists, Ar adsorption isotherms tend to be measured using liquid nitrogen at $77 \mathrm{~K}$, at which the adsorbed phase would be solid ${ }^{[6]}$.

Dubinin's research into activated carbon compared and contrasted $\mathrm{N}_{2}$ adsorption isotherms with $\mathrm{C}_{6} \mathrm{H}_{6}$ adsorption isotherms ${ }^{[10]}$. Benzene offers a slightly larger kinetic diameter, $0.37 \mathrm{~nm}$, a planar structure for probing (deduced) parallel surface or slit-shaped pores, and a chemical structure that would suggest non-polar adsorbate-adsorbent interactions. In fact, its relatively high polarizability acts as a disadvantage if the adsorbent should contain polar surface sites leading to $\pi$-electron, adsorbent surface- 
specific interactions. Benzene's highly carcinogenic properties require appropriate handling procedures.

Micropore size distributions have been defined via DFT models ${ }^{[11]}$, and those based on modifications of the Horvath-Kawazoe models ${ }^{[12,13]}$. Size exclusion or molecular sieve effects have been applied as an alternative hypothesis, exploiting size (and shape) of molecules centred on propane ${ }^{[14]}$. These include propane, iso-butane, and neo-pentane, measured at temperatures in the range 273-298 K. These probes offer dispersion force interactions with an adsorbent and negligible polarizability; their shape differences offer marginal increases in kinetic diameter ${ }^{[14-17]}$. Additional, but infrequently used spherical molecules are $\mathrm{CH}_{4}, \mathrm{CF}_{4}$, and $\mathrm{SF}_{6}$. The chemical conformation of each indicates no dipole moment but the high electronegativity of fluorine could lead to specific interactions with appropriately electron-rich surface sites. Despite this shortcoming, Seaton and co-workers modelled adsorption isotherms of these molecules to define pore network connectivity ${ }^{[18,19]}$.

Considering the advantages and disadvantages of the molecules above, we classify some of them in Table 1 as adsorptives with increasing kinetic diameter, no dipole moment (aside from iso-butane as $0.1 \mathrm{D}^{[20]}$ ), modest polarizability, and subtle shape differences. A comparison of the adsorption isotherms of these adsorptives on the same solid should give qualitative insight into their interaction mechanism with a surface, and provide details of the surface structure and possibly its surface chemistry influence on the isotherm shape. The adsorbent investigated in the work presented below is the previously investigated poly(furfuryl alcohol) (PFFA)-based carbon ${ }^{[21-24]}$. These adsorptives were contacted, compared, and contrasted as high-resolution, averaged isotherms. Linear-linear axis plots helped define Gurvitsch volume results; inconsistency in these and their sources are presented and discussed. The calculated Gurvitsch volumes were compared with those obtained via the DR method. Isotherms also presented as linear-log (relative pressure) axis plots clarified subtle differences in initial uptake processes and their relative pressure ranges, with each interpreted in terms of adsorptive shape, size, and likelihood for adsorbate-adsorbent interaction specificity. This interpretation was further investigated using DR plots to decode fluid-fluid and fluid-solid interactions and pore filling mechanisms. Although all the isotherms showed a Type I shape based on IUPAC classification, our investigation here shows that Type I isotherms can be further classified based on the fluid-fluid and fluid-solid interaction potentials and pore filling mechanism. Although a quite specific adsorbent and adsorptives have been used in this study, the methodologies and principle concepts used here are quite general and represent a foundational development in the topic of adsorption isotherm measurement and analysis.

\section{MATERIALS AND METHODS}

The adsorbent used in this work was a synthetic microporous poly(furfuryl alcohol)-based, activated carbon. Its synthesis and activation procedures were discussed before in detail ${ }^{[22]}$. In summary, distilled 
furfuryl alcohol was mixed with oxalic acid (100:3, w/w) at room temperature. A continuous argon gas flow during mixture polymerisation $\left(150^{\circ} \mathrm{C}\right.$; $\left.1 \mathrm{~h}\right)$ and subsequent carbonization $\left(800{ }^{\circ} \mathrm{C}\right.$; $\left.2 \mathrm{~h}\right)$ resulted in a char. After grinding and sieving $(\approx 100 \mu \mathrm{m})$, the particles were subjected to a repeated, cyclic oxygenargon activation procedure. Firstly, oxygen chemisorption $\left(250^{\circ} \mathrm{C}\right.$; $\left.8 \mathrm{~h}\right)$ followed by desorption and activation under argon atmosphere ( $800{ }^{\circ} \mathrm{C}$; $2 \mathrm{~h}$ ); 9 cycles were determined to be $45 \%$ burn-off. The structural evolution of the adsorbent along the activation pathway has been examined in detail ${ }^{[21]}$, along with the chemistry and surface properties of the sample ${ }^{[24]}$.

Table 1 contains a summary of the physical properties of the non-polar adsorptives used in this study. Each is defined as non-polar with iso-butane (pedantically) the exception, offering a dipole moment of $0.1 \mathrm{D}^{[20]}$. For discussion purposes, we regarded this value as negligible and assumed non-specific interactions with the adsorbent surface.

Table 1 Adsorptives, their physical properties, and conditions used for adsorption experiments

\begin{tabular}{|c|c|c|c|c|c|c|}
\hline Adsorptive & $\begin{array}{l}\text { Molecular } \\
\text { Weight } \\
\text { (kg/kmole) }\end{array}$ & $\begin{array}{c}\text { Temp. (K) / } \\
\text { Temp. } \\
\text { control } \\
\text { device* }\end{array}$ & $\begin{array}{c}\text { Liquid } \\
\text { Density at } \\
\text { Temp. } \\
\left(\mathrm{kg} / \mathrm{m}^{3}\right)^{[25,26]}\end{array}$ & $\begin{array}{c}\text { Minimum } \\
\text { Kinetic } \\
\text { Diameter } \\
(\mathrm{nm})\end{array}$ & $\begin{array}{c}\text { Polarizability } \\
\alpha / 10^{-30}\left(\mathrm{~m}^{3}\right) \\
{ }_{[27]}\end{array}$ & $\begin{array}{c}\text { Grade / } \\
\text { Supplier** }\end{array}$ \\
\hline $\mathrm{CO}_{2}$ & 44.0 & 273 / CIW & 909 & $0.33^{[28]}$ & 2.63 & $\begin{array}{c}>99.999 \% / \\
\text { CG }\end{array}$ \\
\hline Argon (Ar) & 39.9 & $87 / C$ & 1400 & $0.34^{[28]}$ & 1.66 & $\begin{array}{c}>99.999 \% / \\
\text { BOC }\end{array}$ \\
\hline Nitrogen $\left(\mathrm{N}_{2}\right)$ & 28.0 & 77 / LN2 & 808 & $0.36^{[28]}$ & 1.77 & $\begin{array}{c}>99.999 \% / \\
\text { CG }\end{array}$ \\
\hline $\begin{array}{l}\text { Benzene } \\
\left(\mathrm{C}_{6} \mathrm{H}_{6}\right)\end{array}$ & 78.1 & 298 / RC & 879 & $0.37^{[17]}$ & 10.4 & $\begin{array}{c}\text { HPLC Grade } \\
>99.9 \% / \\
\text { SA }\end{array}$ \\
\hline Methane $\left(\mathrm{CH}_{4}\right)$ & 16.0 & $110 / C$ & 422 & $0.38^{[29]}$ & 2.60 & $\begin{array}{c}>99.999 \% / \\
\text { BOC }\end{array}$ \\
\hline${ }^{\dagger}$ iso-butane & 58.1 & 298 / RC & 552 & $0.50^{[6]}$ & - & $\begin{array}{c}>99.995 \% / \\
\text { SA }\end{array}$ \\
\hline $\begin{array}{c}\text { Sulfur- } \\
\text { hexafluoride } \\
\left(\mathrm{SF}_{6}\right)\end{array}$ & 146.1 & 293 / RC & 1450 & $0.55^{[30]}$ & $6.54^{[31]}$ & $\begin{array}{c}>99.75 \% / \\
\text { SA }\end{array}$ \\
\hline
\end{tabular}

* CIW = crushed ice-water bath; C = cryostat; LN2 = liquid nitrogen bath; RC = refrigerated circulator

** CG = Coregas, Australia; BOC = BOC Gases, Australia; SA = Sigma-Aldrich, USA

${ }^{\dagger}$ iso-butane dipole moment $=0.1 \mathrm{D}^{[20]}$

Prior to each experiment, each sample was degassed under the same conditions ( $250^{\circ} \mathrm{C}$; $4 \mathrm{~h} ; 10^{-5} \mathrm{kPa}$ ). $\mathrm{Ar}$ and $\mathrm{CH}_{4}$ gas adsorption isotherms were prepared using an ASAP-2020 apparatus (Micromeritics, Norcross, GA, USA) equipped with a cryostat (Cold Edge Technologies, Allentown, PA, USA). All other adsorption isotherms were obtained using a BELSORP-max gas adsorption apparatus (BEL, Osaka, Japan) equipped with a vapor adsorption kit and a Neslab refrigerated bath circulator controlling temperature $\pm 0.01 \mathrm{~K}$. Helium was used for all dead-space measurements (>99.999\%, ex. BOC Gases, Australia). Repeatability within and reproducibility from different adsorption apparatus indicated self- 
consistent timing for equilibrium penetration of this adsorptive, and apposite removal $\left(25^{\circ} \mathrm{C}\right.$; $1 \mathrm{~h} ; 10^{-5}$ $\mathrm{kPa}$ ) prior to helium dead-space measurements.

As a test of isotherm measurement and reproducibility, each was repeated three times using fresh samples for each experiment. Reproducibility in $\mathrm{N}_{2}$ measurements had been established previously ${ }^{[22]}$. One of the three isotherms for each adsorptive was arbitrarily chosen as the primary and the two repeated isotherms were normalised to it. Separate normalisation of the relative pressures and of the amounts adsorbed was achieved using a MATLAB cubic Hermite interpolation polynomial subroutine. The weighted average values and their uncertainties were developed by propagating the uncertainty in each separate value ${ }^{[32]}$.

\section{RESULTS and DISCUSSION}

The adsorption isotherms and their analyses presented here complement the physical and surface chemistry analyses made previously for this PFFA carbon. Secondly, the obtained results complement those discussed above as molecular sieve analyses. Although the adsorptives used in the current work are classified as non-polar with expected dispersion force interactions with an adsorbent such as the carbon herein, excepting iso-butane, each adsorptive exhibits modest polarizability. Thus, as a set of molecules with increasing kinetic diameter, they would provide adsorption mechanism insight similar to the previously reported non-polar hydrocarbons centred on propane for pore probing and exclusion influences ${ }^{[14-17]}$, and their moderately different polarizability would provide insight into surface chemistry and/or micropore-induced condensation and intramolecular pore packing influences ${ }^{[17]}$.

The classification introduced by Kiselev and used extensively for adsorbate-adsorbent interaction prediction, analysis, (and expectation) is accepted by the present authors ${ }^{[2]}$. In the current work a new perspective is offered giving insight into the mechanisms of adsorption by a wholly microporous adsorbent. This perspective derives from the interpretation of high resolution adsorption isotherms plotted in a non-linear format; logarithm of relative pressure to focus attention on the lowest relative pressure data and their dependent amounts adsorbed.

Prior to making an in-depth discussion of an adsorbent's pore volume it would be necessary to verify if a repeatable basis for the measurements were established, i.e., develop an answer to the question related to helium gas expansion equilibrium and, equally important, if all available pores in the sample were appropriately penetrated by helium at the experimental conditions ${ }^{[8]}$. Several options would be available:

1. Conduct multiple temperature helium expansion measurements on the same equipment on the same sample; 
2. Conduct helium expansion measurements at one temperature on similarly prepared samples on the same equipment with different equilibrium times; or,

3. Make helium expansion on the same samples as 1 and/or 2 above, but on different equipment.

In the current work, $\mathrm{N}_{2}$ adsorption isotherms were prepared following item 2 above: $\mathrm{N}_{2}$ isotherms had been prepared using the BELSORP-max apparatus here, and using the ASAP-2020 apparatus previously ${ }^{[22]}$, giving specific pore volumes as $0.368 \pm 0.015 \mathrm{~cm}^{3}$ (liq)/g via Belsorp and $0.371 \pm 0.005$ $\mathrm{cm}^{3}$ (liq)/g from the ASAP 2020. The consistency between these results suggests that the helium deadspace preparations for the isotherm measurements were repeatable and reproducible.

Fig. 1 shows the adsorption isotherm on a linear $p / p^{o}$ axis for each adsorptive. The amounts adsorbed were converted to $\mathrm{cm}^{3}(\mathrm{liq}) / \mathrm{g}$ using liquid density values at the isotherm measurement temperature (Table 1). By plotting these isotherms as liquid volume adsorbed, one could also deduce the fraction total pore volume filled by each adsorptive relative to the liquid volume $\mathrm{N}_{2}$ adsorbed as the basis ${ }^{[8]}$. The $\mathrm{N}_{2}$ adsorption isotherm in Fig. 1 shows a sharp knee, classified as Type I character and thus one would deduce a relatively narrow micropore size distribution and negligible contribution from mesopores and/or external surface to the amount adsorbed (at pressures $>0.1 p^{0}$ ) ${ }^{[8]}$. The resulting PSD shown in Fig. 2, derived from a QSDFT ${ }^{[11]}$ analysis of the isotherm data, confirms this supposition with a narrow distribution centred at $0.57 \mathrm{~nm}$ and a dispersion of $\approx 0.05 \mathrm{~nm}$. Hu et al. showed repeated $\mathrm{N}_{2}$ adsorption isotherm measurements, and their resulting PSD, on the samples prepared on different occasions were statistically consistent ${ }^{[22]}$. Other studies have confirmed that the calculated PSD shown in Fig. 2 was consistent with those from model-independent methods such as immersion calorimetry and adsorption isosteric heat analyses ${ }^{[15-17]}$. We suggest the PSD shown in Fig. 2 should be regarded as representative and reasonably unequivocal.

The proximity of the selected isotherms to one another questions the statistical significance of their differences in amount adsorbed. Negligible uncertainties for each adsorption isotherm and significant difference between different adsorbates were confirmed by repeated measurements (3-times), as described in the Materials and Methods above. Vertical uncertainty bars are included at medium and high pressures; horizontal uncertainty bars are excluded at high pressures for clarity. The calculated uncertainties for amount adsorbed near saturation were within the ranges reported elsewhere for uncertainty in specific pore volume calculation ${ }^{[33]}$. 
Fig. 1 Adsorption isotherms for the non-polar adsorptives listed in Table 1 on PFA-based microporous carbon samples.

Adsorption isotherm temperatures: $\mathrm{N}_{2}=77 \mathrm{~K} ; \mathrm{CH}_{4}=110 \mathrm{~K} ; \mathrm{Ar}=87 \mathrm{~K} ; \mathrm{C}_{4} \mathrm{H}_{10}$ and $\mathrm{C}_{6} \mathrm{H}_{6}=298 \mathrm{~K} ; \mathrm{SF}_{6}=293 \mathrm{~K} ; \mathrm{CO}_{2}=273 \mathrm{~K}$. 


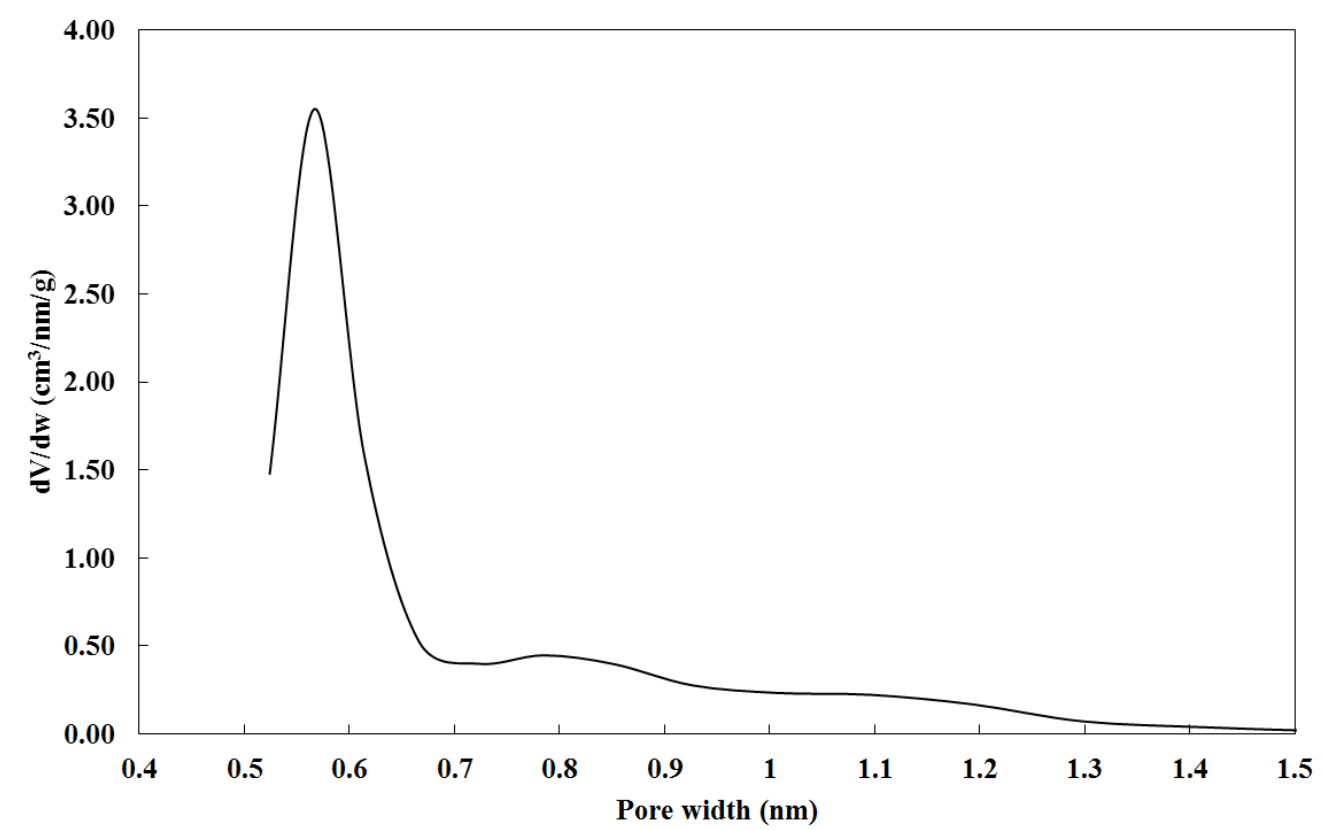

Fig. 2 PFA-based carbon PSD based on QSDFT ${ }^{[11]}$ model on $\mathrm{N}_{2}$ adsorption at 77K

When an adsorption isotherm exhibits or approaches a plateau orthogonal to pressures equivalent to the saturation conditions, the amount adsorbed has been considered equivalent to the maximum specific pore volume available to the adsorptive, $v_{p}$. At such pressures, it has been assumed all pores would be filled with adsorptive and the density of the adsorbed phase would be the density of a continuous liquid phase at the system temperature. This simple approach for pore volume determination was introduced by Gurvitsch and remains an important concept, the Gurvitsch rule ${ }^{[6]}$. Applicability of the rule remains questionable. Some studies suggest a good agreement, regarded as $\pm 5 \%$ difference, between the calculated Gurvitsch volume using different adsorptives in a mesoporous silica-based material ${ }^{[34]}$. The possibility of probe-dependent excluded volume within a micropore distribution could cause each subsequent Gurvitsch volume to differ in value from that established by a basis probe considered to yield a representative pore volume, such as that derived from $\mathrm{N}_{2}$. The excluded volume manifests itself from either of the following criteria:

1- Molecular sieving effect: The adsorptive kinetic diameter exceeds the width of some narrow pores which are then inaccessible to the adsorptive molecule. The volume due to these nonadsorbing, narrower pores would be subtracted from the total pore volume, interpreted as excluded from the calculated Gurvitsch volume;

2- Molecular packing effects governed by a critical ratio of pore size-to-probe size: When the solid contains a narrow distribution of micropores with a mean width close in value to the probe's kinetic diameter, a maximum number of probe-surface contacts would be achieved equivalent to an optimum packing (probe-surface interaction) arrangement. As this ratio increases, molecular packing efficiency reduces to the point where, for an incompressible 
probe, packing inefficiency leads to an increasing free volume within each pore ${ }^{[17]}$. These effects decrease in impact as this ratio exceeds 4 and the Gurvitsch rule becomes independent of molecular packing ${ }^{[17]}$. In partial support of this observation, previous interpretations of molecular packing effects on immersion enthalpy results suggested a criticality of molecular packing effects in the width range $1 / 2$-mean pore width $<$ probe kinetic diameter $<$ mean pore width, i.e. the efficiency of molecular packing effects and its influence on excluded volume may decrease for values of the above ratio $>2$. For samples of this PFA-based carbon, methanol with a kinetic diameter of $0.4 \mathrm{~nm}$ exhibited negligible molecular packing effects, but isopropanol at $0.5 \mathrm{~nm}$ showed marked packing effects, compared with the mean width for this carbon as $0.57 \mathrm{~nm}^{[17]}$.

3- Molecular packing effects dictated by long-range structure development within the densified adsorbed volume: Although the focus of this work is non-polar adsorptives, for completeness of this discussion, hydrogen bonding adsorptives such as water and alcohols may display large decreases in entropy due to adsorption by micropores, creating two and/or three-dimensional adsorbed phase-structure formations equivalent to surface and/or liquid or solid states. The 2D effects would be better classified by criterion 2 above. Since evidence exists suggesting the physical properties of the adsorbed phase of such molecules in micropores are better described by solid-phase than liquid phase ${ }^{[35-37]}$, the apparent excluded volume may also be due to an incorrect density attributed to the adsorbed phase.

The plateau amount adsorbed for each adsorptive in Fig 1 was extrapolated linearly to $p \approx p^{o}$, and the corresponding Gurvitsch volume plotted as a function of probe kinetic diameter in Fig. 3. 
Fig. 3 Calculated Gurvitsch volume for weak fluid-fluid interaction probes as a function of their kinetic diameter. Weighted average Gurvitsch volume (-); weighted standard deviation of the average Gurvitsch volume (---)

The Gurvitsch volumes for $\mathrm{CO}_{2}$, iso-butane, and $\mathrm{SF}_{6}$ were not reported due to experimental equipment limitations. The saturation vapour pressure of each at the measurement temperature was $>>1$ bar; the sample holder was glass.

From the details in Table 1 and the lower limit of the $\mathrm{N}_{2}$ PSD defined in Fig. 2, it is clear $\mathrm{Ar}, \mathrm{C}_{6} \mathrm{H}_{6}$, and $\mathrm{CH}_{4}$ were sufficiently small $(<0.39 \mathrm{~nm})$ to penetrate and be adsorbed by all available pores. This conclusion supports our previous immersion calorimetry analyses which indicated no molecular packing effects for probes with similarly small dimensions ${ }^{[17]}$. These adsorptives were independent of criterion 1 and 2, and, since they contain no hydrogen bonding capability they were also independent of criterion 3, leading to relatively consistent Gurvitsch volumes with average value $0.368 \pm 0.015$ $\mathrm{cm}^{3}(\mathrm{liq}) / \mathrm{g}$, equivalent to $4.11 \%$ relative uncertainty. Both this Gurvitsch volume and its uncertainty were evaluated as weighted averages which included the uncertainty in each value and propagated to the value presented ${ }^{[32]}$. This relative uncertainty is consistent with observations reported for mesoporous silica-based adsorbents as $\pm 5 \%{ }^{[34]}$. Since the Gurvitsch volumes for this set of adsorptives were independent of criteria 1-3, we suggest the average value should be regarded as a representative value for total pore volume of this adsorbent.

The well-known DR equation ${ }^{[38]}$ has been frequently used in the literature for analysis and interpretation of the mechanism of the micropore filling ${ }^{[39-42]}$ :

$$
\log \left(V_{a d s}\right)=\log \left(V_{\text {mic }}\right)-D \log ^{2}\left(p^{o} / p\right)
$$

where $V_{\text {mic }}$ is the total micropore volume and $D$ is an empirical constant related to the characteristic free energy of adsorption. A plot of Eq. [1] provides a linear range from which the micropore volume might be evaluated; values for each adsorptive are reproduced in Table 2, along with Gurvitsch volumes. The DR plot for each adsorptive in Table 1 is also shown in Fig. 4. Uncertainties in the DR data were propagated from standard deviations in isotherm data and shown as bars in Fig. $4^{[43]}$. The uncertainty in the subsequent micropore volumes were evaluated via weighted mean least squares methods ${ }^{\text {[32] }}$.

Table 2 Calculated pore volumes via DR method and comparison with Gurvitsch volume

\begin{tabular}{|c|c|c|}
\hline Adsorptive & Gurvitsch Volume $\left(\mathrm{cm}^{3} / \mathrm{g}\right)$ & Micropore volume from DR analysis $\left(\mathrm{cm}^{3} / \mathrm{g}\right)^{*}$ \\
\hline $\mathrm{CO}_{2}$ & - & 0.339 \\
\hline Argon $(\mathrm{Ar})$ & $0.356 \pm 0.004$ & 0.350 \\
\hline Nitrogen $\left(\mathrm{N}_{2}\right)$ & $0.388 \pm 0.013$ & 0.360 \\
\hline Benzene $\left(\mathrm{C}_{6} \mathrm{H}_{6}\right)$ & $0.356 \pm 0.005$ & 0.343 \\
\hline
\end{tabular}




\begin{tabular}{|c|c|c|}
\hline Methane $\left(\mathrm{CH}_{4}\right)$ & $0.371 \pm 0.002$ & 0.360 \\
\hline iso-butane & - & 0.347 \\
\hline Sulfur-hexafluoride $\left(\mathrm{SF}_{6}\right)$ & - & 0.360 \\
\hline
\end{tabular}

* The evaluated relative uncertainties for the data were exceptionally small. The average relative uncertainty was $(3.6 \pm 1.7) \times 10^{-5} \mathrm{~cm}^{3} / \mathrm{g}$

Table 2 clearly indicates a reasonable agreement between DR-method derived pore volumes and the Gurvitsch volumes for most of the adsorptives. This good agreement is due to the fact that the shape of the adsorption isotherm would be fitted by the assumptions underpinning the TVFM ${ }^{[44]}$. The DR plots shown in Fig. 4 exemplify the flexibility of the method to evaluate micropore volumes for incomplete adsorption isotherms, such as those for $\mathrm{CO}_{2}, \mathrm{SF}_{6}$, and iso-butane due to the (equipment-imposed) maximum pressure limitations.

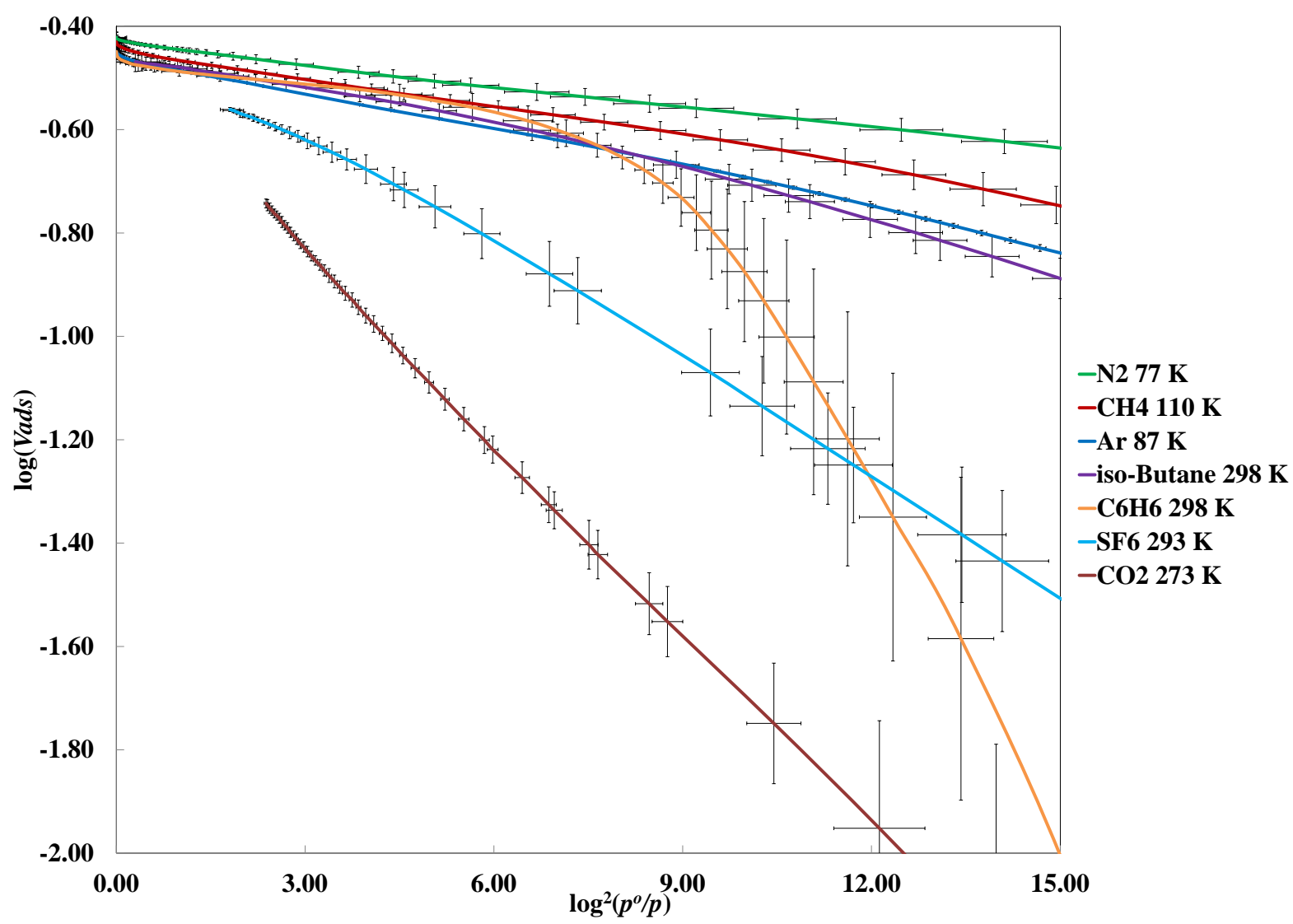

Fig. 4 Linearized DR plots for the adsorptives listed in Table 1 
Fig. 5 Log-scale adsorption isotherms for the non-polar adsorptives listed in Table 1 on PFA-based microporous carbon samples. Uptake relative pressure ranges: early uptake: $1 \times 10^{-8}-1 \times 10^{-4}$; medium uptake: $1 \times 10^{-4}-1 \times 10^{-2}$; late uptake: $1 \times 10^{-2}-1$ 
Fig. 5 shows the adsorption isotherms as a log-scale $p / p^{o}$ abscissa, amplifying the lower relative pressure influence on amounts adsorbed for each adsorptive. For comparison with Fig. 1, the ordinate axis remained amount adsorbed with units as $\mathrm{cm}^{3}(\mathrm{liq}) / \mathrm{g}$. Since these measurements were made using the manometric adsorption method (previously regarded as volumetric adsorption), the uncertainty in the amounts adsorbed would increase with each subsequent pressure-amount adsorbed (pair) calculation. The uncertainties in the amounts adsorbed and in the relative pressures were developed as described above. Pressure gauge sensitivity, thermal control of the dosing volume, and control of the liquid nitrogen level contribute to the uncertainty in the amount adsorbed ${ }^{[45]}$. Pressure gauge sensitivity contributes largest at the limits of lower pressure hence, the uncertainty in the average values decreased with increasing pressure and associated amounts adsorbed. The plot format provides scope for a qualitative analysis of the relative differences in mode of adsorption and associated differences in adsorption energetics for each adsorptive.

Each isotherm exhibited different minimum relative pressures above which reliably measureable uptake occurred, after which its shape became convex to the pressure axis, interpreted as adsorption and pore filling as condensation in the narrowest accessible micropores. This condensation was associated with micropore filling with increasing relative pressure and concave shape towards equivalence to the probedependent Gurvitsch volume. For each adsorptive, attention should also be drawn to each linear section in the DR plot in Fig. 4 which corresponded to the condensation step equivalent to micropore filling. Adsorption post pore filling on available external surface, was indicated in Fig. 5 by additional convex shape change in the overall uptake curves, typically at the upper limits of pressures for these Type I isotherms. Generally, the non-polar adsorptives $\left(\mathrm{N}_{2}, \mathrm{Ar}\right.$, and $\left.\mathrm{CH}_{4}\right)$, characterised as cryogenic fixed gases with relatively weak fluid-fluid interactions, exhibited uptake as adsorption by the solid surface with simultaneous fluid condensation. The isotherm shape typically expands across the pressure range, with uptake increasing steadily with increasing equilibrium relative pressure. This micropore filling mechanism would be equivalent to the linear range shown in Fig. 4, again over a relatively broad range of relative pressure. In contrast, the adsorptives showing stronger fluid-fluid interactions either due to quadrupole interactions $\left(\mathrm{C}_{6} \mathrm{H}_{6}\right.$ and $\left.\mathrm{CO}_{2}\right)$ and/or high polarizability $\left(\mathrm{C}_{6} \mathrm{H}_{6}\right.$ and $\left.\mathrm{SF}_{6}\right)$, exhibited uptake interpreted as fluid-fluid interaction-supported (pore filling) condensation within the pore structure. For the latter category, the isotherm shape showed a sharp increase in uptake over a relatively narrow pressure range, followed by a "knee" then a distinct plateau. This S-shape in the isotherm indicated contributions from specific fluid-fluid and fluid-solid interactions, and equated with specific interactions resulting in deviations from linearity in the DR plots (Fig. 4). This adsorption characteristic would be better exemplified by polar adsorptives ${ }^{[3]}$.

The isotherms in Fig. 5, and adsorption isotherms in general, could be refined and analysed by classifying them into relative pressure ranges associated with the majority of amount adsorbed, leading to the pore filling condensation mechanism: early, $p / p^{o}=10^{-8}-10^{-4}$; medium, $p / p^{o}=10^{-4}-10^{-2}$; and late, 
$p / p^{o}=10^{-2}-10^{0}(=1)$. These ranges accommodate adsorption via specific interaction forces or nonspecific interaction forces associated with adsorptive polarity and/or polarizability, and account for adsorptive molecular shape. The ranges would be equally applicable to polar adsorptives of different sizes and shapes ${ }^{[3]}$. The isotherms in this work were classified further below:

a) Non-polar adsorptives with primarily non-specific interactions: Adsorptives in this class interact with adsorbents via a relatively moderate fluid-solid interaction energy, up to 2-3 times latent heat of adsorptive condensation ${ }^{[46]}$, and subsequent adsorption enthalpy supported by relatively small fluid-fluid interactions as part of the condensation process either within pore filling or adsorbed layer development. Examples include $\mathrm{Ar}, \mathrm{N}_{2}, \mathrm{CH}_{4}$ and iso-butane. The adsorption mechanism would be a "layering" process where the smallest pores, equivalent in width to the adsorptive's kinetic diameter, incite maximum adsorbate-surface contact as layering along the axis of the pore, if it were a slit (or cylinder), followed by increasing fluidfluid interactions in this plane, resulting in pore filling. A similar type of statistical layering could be envisaged in wider pores leading to multiple layer adsorption with diminishing fluidsolid contributions to the increasing fluid-fluid interactions within subsequent adsorbed "layers".

For this class of adsorptives, the low pressure adsorption isotherms in Fig. 5 were quite illustrative. At the lowest equilibrium pressures, adsorption occurred at or in vacant sites as pores within the structure wherein fluid-solid interactions predominated. Once multiple layers were formed, fluid-solid interactions diminished at the expense of (increasing) fluid-fluid interactions. Since this transition was gradual, no sudden change occurred in the adsorption isotherm shape with increasing pressure; neither interaction impacted on the isotherm shape. The amount adsorbed increased gradually with increasing $p / p^{o}$ and the uptake was relatively expansive. For this class of adsorptives, the pore filling as a "layering” process indicating nonspecific adsorption mechanism was also identified in Fig. 4 by the linear DR plots extended over a broad range of relative pressures.

b) Non-polar adsorptives with potential for specific interaction: Adsorptives in this class have electronic structures promoting localisation when in the adsorbed state as specific interactions due to quadrupole moment and/or $\pi$-electron distributions. Examples include $\mathrm{CO}_{2}$ and $\mathrm{C}_{6} \mathrm{H}_{6}$. In the gas phase, these adsorptives tend to be non-polar, however, the data in Table 1 indicate both are polarizable, leading to a quadrupole moment in $\mathrm{CO}_{2}$ and due to $\pi$-electrons in $\mathrm{C}_{6} \mathrm{H}_{6}$, supporting the capacity for specific interactions. These intramolecular forces also produce substantial fluid-fluid interaction contributions within the adsorbed phase. Consequently, such adsorptives exhibit pore filling as a combination of cluster formation and layering mechanisms, exemplified as medium pressure range condensation within pores. 
The low pressure adsorption isotherms for this class of adsorptives were also shown in Fig. 5. The isotherms showed higher relative pressures prior to measurably reproducible uptake than those in Category (a). Pore filling occurred over a narrow relative pressure range induced by strong intramolecular interactions in the adsorbed phase. Although the details for $\mathrm{CO}_{2}$ were incomplete due to experimental limitations, $\mathrm{C}_{6} \mathrm{H}_{6}$ exhibited complete pore filling to give pore volumes equivalent to the basis adsorptive, $\mathrm{N}_{2}$.

Specific interaction potentials, cluster formation, and large intramolecular forces were identified as the principal reasons for non-linear DR plots ${ }^{[47]}$. Although $\mathrm{CO}_{2}$ and $\mathrm{C}_{6} \mathrm{H}_{6}$ are classified as non-polar adsorptives, due to their quadrupole moment and $\pi$-electron interactions, they have the potential for specific interactions. Such specific interactions resulted in nonlinearity in the DR plots clearly identified in Fig. 4 for benzene. For $\mathrm{CO}_{2}$, this non-linearity would have been exhibited at higher relative pressures if such measurements had been experimentally achievable.

c) Halogenated adsorptives: As a class of adsorptives, these are infrequently investigated. As Group 17 elements they possess considerable electronegativity, and when bound to other atoms could promote specific interactions with adsorbent surfaces. The example used here was $\mathrm{SF}_{6}$. Table 1 indicates this adsorptive was essentially non-polar, but the presence of hecta-poles within its structure underpinned why it would be intermediate $\mathrm{CO}_{2}$ and $\mathrm{C}_{6} \mathrm{H}_{6}$ in its ability to be polarised, possibly in the presence of high energy surface sites ${ }^{[48]}$. The adsorption isotherm for this molecule, shown in Fig. 5, exhibited an early uptake similar to the other non-polar adsorptives (as Category (a) above), however the strongly electronegative feature of the fluorine atoms induced specific interactions as localised (specific) adsorption polarisation, similar to interactions available for $\mathrm{N}_{2}$ and iso-butane. However, unlike the non-polar adsorptives, the $\mathrm{SF}_{6}$ isotherm exhibited a considerably narrower range of relative pressuredependent uptake extending across the medium and late pressure ranges, synonymous with increasing fluid-fluid interactions within the condensed phase of the larger micropores. The modest pressure range during condensation was probably indicative of its capacity to probe efficiently the wider micropores within their distribution.

The electronegativity of the fluorine atom and the large polarizability of $\mathrm{SF}_{6}$ molecule resulted in localized specific interactions and relatively large intramolecular interactions; consequently, a relatively large departure from linearity in DR plot was found for this adsorptive. This nonlinearity would be even more pronounced if higher pressure data were available with the current adsorption apparatus.

\section{CONCLUSIONS}


Repeated and controlled adsorption isotherms of selected frequently-used adsorptives were reported and compared quantitatively for pore volume assessment and qualitatively for adsorption mechanism determination. The most important conclusions are:

- Very low pressure adsorption data examined via a log-axis relative pressure, indicated isotherm reproducibility with negligible uncertainty in the amount adsorbed.

- Non-polar adsorptives with kinetic diameter less than the bulk of the micropore width, regardless of adsorptive shape, and offering no possibility of hydrogen bonding or specific interaction with the adsorbent, would give narrow levels of equivalence in Gurvitsch volume.

- Qualitatively, the mechanism of adsorption depends on the combination of adsorbate-adsorbate and adsorbate-adsorbent interactions. From the list of adsorptives presented herein, isotherms could be classified as follows:

a- Non-polar adsorptives with non-specific interactions ( $\mathrm{Ar}, \mathrm{N}_{2}, \mathrm{CH}_{4}$ and iso-butane); the adsorption mechanism began as fluid-solid interactions followed by a layering effect involving fluid condensation. The uptake in the isotherm started at the lowest measureable pressures, gradually extending through medium-valued and up to the highest measured pressures. DR plots for this class of adsorptives exhibited a linear behaviour over a wide relative pressure range, indicating layering and pore filling condensation adsorption mechanisms.

b- Non-polar adsorptives with potential for specific interactions $\left(\mathrm{CO}_{2}\right.$ and $\left.\mathrm{C}_{6} \mathrm{H}_{6}\right)$; Adsorptives such as these were not polar but presented possible quadrupole interactions with any relatively high or polarisable surface sites leading to cluster formation and condensation, and eventually layering due to their moderate-level fluid-fluid interactions. The uptake in the isotherm started in the medium-range relative pressures followed by relatively sharp increase in amount adsorbed as condensation pore filling. This combined cluster formation, condensation, and layering mechanism resulted in deviations from linearity in DR plots.

c- Halogenated adsorptives $\left(\mathrm{SF}_{6}\right)$; such adsorptives would be neither polar nor contain quadrupole moments, however, they could be polarizable, promoting early uptake at lowest measureable pressures. The strongly electronegative halogen atoms promoted specific interactions at these lowest pressures, followed by pore filling condensation over a relatively narrow pressure range, extending up to the highest measureable pressures. The overall shape due this mechanism was S-shaped. The relatively large polarizability of this adsorptive and the electronegativity of the fluorine atoms resulted in specific interactions with polar sites on the solid, and these relatively strong intramolecular interactions were the primary contributions to non-linear DR plots. 


\section{ACKNOWLEDGEMENTS}

The authors thank the Australian Research Council discovery program (DP110101293) for funding support and S.H.M also thanks the University of South Australia for a postgraduate research scholarship.

\section{REFERENCES}

[1] S. J. Gregg, K. S. W. Sing, Adsorption, Surface Area and Porosity, Academic Press, London, 1967.

[2] A. V. Kiselev J. Colloid Interface Sci. 1968, 28, 430-442.

[3] S. H. Madani, M. J. Biggs, F. Rodrígues-Reinoso, P. Pendleton Submitted to ChemPhysChem. 2017.

[4] K. S. W. Sing Chem. Ind. (London). 1968, 44, 1520-1521.

[5] J. Landers, G. Y. Gor, A. V. Neimark Colloid. Surface. A. 2013, 437, 3-32.

[6] F. Rouquerol, J. Rouquerol, K. Sing, Adsorption by powders and porous solids Academic Press, London, 1999.

[7] H. Marsh, F. Rodríguez-Reinoso, Activated Carbon, Elsevier Science, 2006.

[8] M. Thommes, K. Kaneko, V. Neimark Alexander, P. Olivier James, F. Rodríguez-Reinoso, J.

Rouquerol, S. W. Sing Kenneth Pure Appl. Chem. 2015, 87, 1051.

[9] K. A. Cychosz, R. Guillet-Nicolas, J. Garcia-Martinez, M. Thommes Chem. Soc. Rev. 2017, 46, 389414.

[10] M. M. Dubinin, E. F. Polstyanov Izv. Akad. Nauk, Ser. Khim. 1966, 793-801.

[11] A. V. Neimark, Y. Lin, P. I. Ravikovitch, M. Thommes Carbon. 2009, 47, 1617-1628.

[12] G. Horvath, K. Kawazoe J. Chem. Eng. Jpn. 1983, 16, 470.

[13] S. U. Rege, R. T. Yang AlChE J. 2000, 46, 734-750.

[14] P. J. M. Carrott, R. A. Roberts, K. S. W. Sing in A new method for the determination of micropore size distributions, Vol. (Ed.^Eds.: Editor), Elsevier, City, 1988, pp.89-100.

[15] S. H. Madani, C. Hu, A. Silvestre-Albero, M. J. Biggs, F. Rodríguez-Reinoso, P. Pendleton Carbon. 2016, 96, 1106-1113.

[16] S. H. Madani, S. Sedghi, M. J. Biggs, P. Pendleton ChemPhysChem. 2015, 16, 3797-3805.

[17] S. H. Madani, A. Silvestre-Albero, M. J. Biggs, F. Rodríguez-Reinoso, P. Pendleton

ChemPhysChem. 2015, 16, 3984-3991.

[18] M. V. López-Ramón, J. Jagiełło, T. J. Bandosz, N. A. Seaton Langmuir. 1997, 13, 4435-4445.

[19] Q. Cai, A. Buts, M. J. Biggs, N. A. Seaton Langmuir. 2007, 23, 8430-8440.

[20] B. Poling, J. Prausnitz, J. O. Connell, The Properties of Gases and Liquids, McGraw-Hill Education, 2000.

[21] C. Hu, A. C. Y. Liu, M. Weyland, S. H. Madani, P. Pendleton, F. Rodríguez-Reinoso, K. Kaneko, M. J. Biggs Carbon. 2015, 85, 119-134.

[22] C. Hu, S. Sedghi, S. H. Madani, A. Silvestre-Albero, H. Sakamoto, P. Kwong, P. Pendleton, R. J. Smernik, F. Rodríguez-Reinoso, K. Kaneko, M. J. Biggs Carbon. 2014, 78, 113-120.

[23] C. Hu, S. Sedghi, A. Silvestre-Albero, G. G. Andersson, A. Sharma, P. Pendleton, F. RodríguezReinoso, K. Kaneko, M. J. Biggs Carbon. 2015, 85, 147-158.

[24] S. Sedghi, S. H. Madani, C. Hu, A. Silvestre-Albero, W. Skinner, P. Kwong, P. Pendleton, R. J.

Smernik, F. Rodríguez-Reinoso, M. J. Biggs Carbon. 2015, 95, 144-149.

[25] C. F. Spencer, R. P. Danner J. Chem. Eng. Data. 1972, 17, 236-241.

[26] R. H. Perry, D. W. Green, Perry's chemical engineer's handbook, McGraw-Hill, New York, 1999.

[27] P. Atkins, J. d. Paula, Physical Chemistry, Oxford Press, Oxford, 2002.

[28] K. S. W. Sing in 10 - Adsorption by Active Carbons, Vol. (Eds.: F. Rouquerol, J. Rouquerol, K. S. W.

Sing, P. Llewellyn, G. Maurin), Academic Press, Oxford, 2014, pp.321-391.

[29] N. Mehio, S. Dai, D.-e. Jiang J. Phys. Chem. A. 2014, 118, 1150-1154.

[30] P. Dedek, A. S. Webber, V. Janout, R. A. Hendel, S. L. Regen Langmuir. 1994, 10, 3943-3945. 
[31] L. W. Beegle, I. Kanik, L. Matz, H. H. Hill Jr Int. J. Mass Spectrom. 2002, 216, 257-268.

[32] J. R. Taylor, An Introduction to Error Analysis: The Study of Uncertainties in Physical Measurements, University Science Books, New York, 1982.

[33] A. Badalyan, P. Pendleton J. Colloid Interface Sci. 2008, 326, 1-7.

[34] D. W. McKee J. Phys. Chem. 1959, 63, 1256-1259.

[35] M. Thommes, C. Morlay, R. Ahmad, J. P. Joly Adsorption. 2011, 17, 653.

[36] R. S. Vartapetyan, A. M. Voloshchuk, A. K. Buryak, C. D. Artamonova, R. L. Belford, P. J. Ceroke, D. V. Kholine, R. B. Clarkson, B. M. Odintsov Carbon. 2005, 43, 2152-2159.

[37] T. liyama, M. Ruike, K. Kaneko Chem. Phys. Lett. 2000, 331, 359-364.

[38] M. M. Dubinin, L. V. Radushkevich Dokl. Akad. Nauk. 1947, 55, 331.

[39] A. Andreu, H. F. Stoeckli, R. H. Bradley Carbon. 2007, 45, 1854-1864.

[40] M. M. Dubinin, H. F. Stoeckli J. Colloid Interface Sci. 1980, 75, 34.

[41] U. Huber, H. F. Stoeckli, J. P. Houriet J. Colloid Interface Sci. 1978, 67, 195.

[42] F. Stoeckli, A. Guillot, A. M. Slashi, D. Hugi-Cleary Carbon. 2002, 40, 383-388.

[43] P. Pendleton, A. Badalyan Colloids Surf., A: Physicochem. Eng. Asp. 2007, 300, 253-258.

[44] M. M. Dubinin in Physical adsorption of gases and vapors in micropores, Vol. 9 (Ed. D. A.

Cadenhead), Academic Press, New York, 1975, pp.1-70.

[45] A. Badalyan, P. Pendleton Langmuir. 2003, 19, 7919-7928.

[46] D. H. Everett, J. C. Powl J. Chem. Soc., Faraday Trans. 1. 1976, 72, 619.

[47] F. Rodríguez-Reinoso, M. Molina-Sabio, M. A. Munecas J. Phys. Chem. 1992, 96, 2707-2713.

[48] G. Maroulis Chem. Phys. Lett. 1999, 312, 255-261. 